\title{
Measurement of the resonant and nonresonant branching ratios in $\Xi_{c}^{0} \rightarrow \Xi^{0} K^{+} K^{-}$
}

J. T. McNeilø, ${ }^{9}$ J. Yelton, ${ }^{9}$ J. Bennett, ${ }^{49}$ I. Adachi, ${ }^{16,12}$ K. Adamczyk, ${ }^{57}$ J. K. Ahn, ${ }^{37}$ H. Aihara, ${ }^{80}$ S. Al Said, ${ }^{74,34}$ D. M. Asner, ${ }^{3}$ H. Atmacan, ${ }^{7}$ V. Aulchenko, ${ }^{4,60}$ T. Aushev, ${ }^{18}$ R. Ayad ${ }^{74}$ V. Babu, ${ }^{8}$ S. Bahinipati, ${ }^{21}$ P. Behera, ${ }^{23}$ M. Bessner, ${ }^{15}$ T. Bilka, ${ }^{5}$ J. Biswal, ${ }^{31}$ A. Bobrov, ${ }^{4,60}$ G. Bonvicini, ${ }^{85}$ A. Bozek, ${ }^{57}$ M. Bračko, ${ }^{46,31}$ T. E. Browder, ${ }^{15}$ M. Campajola, ${ }^{28,52}$ L. Cao, ${ }^{2}$ D. Červenkov, ${ }^{5}$ P. Chang, ${ }^{56}$ V. Chekelian, ${ }^{47}$ A. Chen, ${ }^{54}$ B. G. Cheon, ${ }^{14}$ K. Chilikin, ${ }^{41}$ K. Cho ${ }^{36}$ S.-J. Cho, ${ }^{87}$ S.-K. Choi ${ }^{13}$ Y. Choi,${ }^{72}$ S. Choudhury, ${ }^{22}$ D. Cinabro, ${ }^{85}$ S. Cunliffe, ${ }^{8}$ S. Das, ${ }^{45}$ N. Dash,${ }^{23}$ G. De Nardo, ${ }^{28,52}$ R. Dhamija, ${ }^{22}$ F. Di Capua, ${ }^{28,52}$ Z. Doležal, ${ }^{5}$ T. V. Dong, ${ }^{10}$ S. Eidelman, ${ }^{4,60,41}$ T. Ferber, ${ }^{8}$ B. G. Fulsom, ${ }^{62}$ V. Gaur, ${ }^{84}$ N. Gabyshev, ${ }^{4,60}$ A. Garmash, ${ }^{4,60}$ A. Giri, ${ }^{22}$ P. Goldenzweig, ${ }^{32}$ B. Golob, ${ }^{42,31}$ O. Grzymkowska, ${ }^{57}$ Y. Guan, ${ }^{7}$ K. Gudkova, ${ }^{4,60}$ C. Hadjivasiliou, ${ }^{62}$ K. Hayasaka, ${ }^{59}$ H. Hayashii, ${ }^{53}$ W.-S. Hou ${ }^{56}$ C.-L. Hsu, ${ }^{73}$ K. Huang, ${ }^{56}$ K. Inami, ${ }^{51}$ A. Ishikawa, ${ }^{16,12}$ R. Itoh, ${ }^{16,12}$ M. Iwasaki ${ }^{61}$ W. W. Jacobs,${ }^{24}$ H. B. Jeon, ${ }^{39}$ S. Jia, ${ }^{10}$ Y. Jin, ${ }^{80}$ K. K. Joo, ${ }^{6}$ A. B. Kaliyar, ${ }^{75}$ K. H. Kang, ${ }^{39}$ G. Karyan, ${ }^{8}$ H. Kichimi, ${ }^{16}$ B. H. Kim ${ }^{68}$ C. H. Kim, ${ }^{14}$ D. Y. Kim, ${ }^{71}$ S. H. Kim ${ }^{68}$ Y.-K. Kim,${ }^{87}$ K. Kinoshita, ${ }^{7}$ P. Kodyšs ${ }^{5}$ T. Konno, ${ }^{35}$ S. Korpar, ${ }^{46,31}$ P. Križan, ${ }^{42,31}$ R. Kroeger, ${ }^{49}$ P. Krokovny, ${ }^{4,60}$ T. Kuhr, ${ }^{43}$ R. Kulasiri, ${ }^{33}$ M. Kumar, ${ }^{45}$ K. Kumara ${ }^{85}$ Y.-J. Kwon, ${ }^{87}$ K. Lalwani, ${ }^{45}$ S. C. Lee,${ }^{39}$ J. Li, ${ }^{39}$ L. K. Li, ${ }^{7}$ Y. B. Li ${ }^{63}$ L. Li Gioi, ${ }^{47}$ J. Libby, ${ }^{23}$ K. Lieret, ${ }^{43}$ Z. Liptak, ${ }^{15},{ }^{*}$ D. Liventsev, ${ }^{85,16}$ C. MacQueen, ${ }^{48}$ M. Masuda ${ }^{79,65}$ T. Matsuda ${ }^{50}$ D. Matvienko, ${ }^{4,60,41}$ M. Merola, ${ }^{28,52}$ F. Metzner, ${ }^{32}$ K. Miyabayashi, ${ }^{53}$ R. Mizuk, ${ }^{41,18}$ G. B. Mohanty ${ }^{75}$ S. Mohanty,${ }^{75,83}$ T. J. Moon, ${ }^{68}$ R. Mussa, ${ }^{29}$ M. Nakao, ${ }^{16,12}$ A. Natochii, ${ }^{15}$ L. Nayak ${ }^{22}$ M. Nayak ${ }^{77}$ M. Niiyama ${ }^{38}$ N. K. Nisar, ${ }^{3}$ S. Nishida, ${ }^{16,12}$ K. Ogawa, ${ }^{59}$ S. Ogawa, ${ }^{78}$ H. Ono, ${ }^{58,59}$ Y. Onuki, ${ }^{80}$ P. Oskin, ${ }^{41}$ G. Pakhlova, ${ }^{18,41}$ S. Pardi, ${ }^{28}$ H. Park, ${ }^{39}$ S.-H. Park, ${ }^{87}$ S. Paul, ${ }^{76,47}$ T. K. Pedlar, ${ }^{44}$ R. Pestotnik,,${ }^{31}$ L. E. Piilonen, ${ }^{84}$ T. Podobnik, ${ }^{42,31}$ V. Popov,${ }^{18}$ E. Prencipe,${ }^{19}$ M. T. Prim, ${ }^{32}$ M. Röhrken, ${ }^{8}$ A. Rostomyan, ${ }^{8}$ N. Rout, ${ }^{23}$ G. Russo, ${ }^{52}$ D. Sahoo, ${ }^{75}$ Y. Sakai, ${ }^{16,12}$ S. Sandilya, ${ }^{22}$ A. Sangal,${ }^{7}$ L. Santelj, ${ }^{42,31}$ V. Savinov, ${ }^{64}$ G. Schnell,,${ }^{1,20}$ J. Schueler, ${ }^{15}$ C. Schwanda, ${ }^{26}$ Y. Seino ${ }^{59}$ K. Senyo, ${ }^{86}$ M. E. Sevior, ${ }^{48}$ M. Shapkin, ${ }^{27}$ C. Sharma ${ }^{45}$ V. Shebalin, ${ }^{15}$ J.-G. Shiu ${ }^{56}$ F. Simon, ${ }^{47}$ E. Solovieva, ${ }^{41}$ M. Starič ${ }^{31}$ Z. S. Stottler, ${ }^{84}$ J. F. Strube, ${ }^{62}$ M. Sumihama, ${ }^{11}$ T. Sumiyoshi, ${ }^{82}$ M. Takizawa, ${ }^{69,17,66}$ U. Tamponi, ${ }^{29}$ K. Tanida ${ }^{30}$ Y. Tao, ${ }^{9}$ F. Tenchini, ${ }^{8}$ M. Uchida, ${ }^{81}$ S. Uehara, ${ }^{16,12}$ Y. Unno, ${ }^{14}$ S. Uno ${ }^{16,12}$ R. Van Tonder, ${ }^{2}$ G. Varner, ${ }^{15}$ A. Vinokurova, ${ }^{4,60}$ V. Vorobyev, ${ }^{4,60,41}$ C. H. Wang, ${ }^{55}$ E. Wang, ${ }^{64}$ M.-Z. Wang, ${ }^{56}$ P. Wang, ${ }^{25}$ M. Watanabe, ${ }^{59}$ S. Watanuki, ${ }^{40}$ E. Won, ${ }^{37}$ X. Xu, ${ }^{70}$ B. D. Yabsley, ${ }^{73}$ W. Yan, ${ }^{67}$ H. Ye, ${ }^{8}$ J. H. Yin, ${ }^{37}$ Z. P. Zhang, ${ }^{67}$ V. Zhilich, ${ }^{4,60}$ and V. Zhukova ${ }^{41}$

(The Belle Collaboration)

\author{
${ }^{1}$ University of the Basque Country UPV/EHU, 48080 Bilbao, Spain \\ ${ }^{2}$ University of Bonn, 53115 Bonn, Germany \\ ${ }^{3}$ Brookhaven National Laboratory, Upton, New York 11973, USA \\ ${ }^{4}$ Budker Institute of Nuclear Physics SB RAS, Novosibirsk 630090, Russia \\ ${ }^{5}$ Faculty of Mathematics and Physics, Charles University, 12116 Prague, Czech Republic \\ ${ }^{6}$ Chonnam National University, Gwangju 61186, South Korea \\ ${ }^{7}$ University of Cincinnati, Cincinnati, Ohio 45221, USA \\ ${ }^{8}$ Deutsches Elektronen-Synchrotron, 22607 Hamburg, Germany \\ ${ }^{9}$ University of Florida, Gainesville, Florida 32611, USA \\ ${ }^{10}$ Key Laboratory of Nuclear Physics and Ion-beam Application (MOE) and Institute of Modern Physics, \\ Fudan University, Shanghai 200443, China \\ ${ }^{11}$ Gifu University, Gifu 501-1193, Japan \\ ${ }^{12}$ SOKENDAI (The Graduate University for Advanced Studies), Hayama 240-0193, Japan \\ ${ }^{13}$ Gyeongsang National University, Jinju 52828, South Korea \\ ${ }^{14}$ Department of Physics and Institute of Natural Sciences, Hanyang University, Seoul 04763, South Korea \\ ${ }^{15}$ University of Hawaii, Honolulu, Hawaii 96822, USA \\ ${ }^{16}$ High Energy Accelerator Research Organization (KEK), Tsukuba 305-0801, Japan \\ ${ }^{17}$ J-PARC Branch, KEK Theory Center, High Energy Accelerator Research Organization (KEK), Tsukuba \\ 305-0801, Japan \\ ${ }^{18}$ Higher School of Economics (HSE), Moscow 101000, Russia \\ ${ }^{19}$ Forschungszentrum Jülich, 52425 Jülich, Germany \\ ${ }^{20}$ IKERBASQUE, Basque Foundation for Science, 48013 Bilbao, Spain \\ ${ }^{21}$ Indian Institute of Technology Bhubaneswar, Satya Nagar 751007, India \\ ${ }^{22}$ Indian Institute of Technology Hyderabad, Telangana 502285, India \\ ${ }^{23}$ Indian Institute of Technology Madras, Chennai 600036, India \\ ${ }^{24}$ Indiana University, Bloomington, Indiana 47408, USA
}




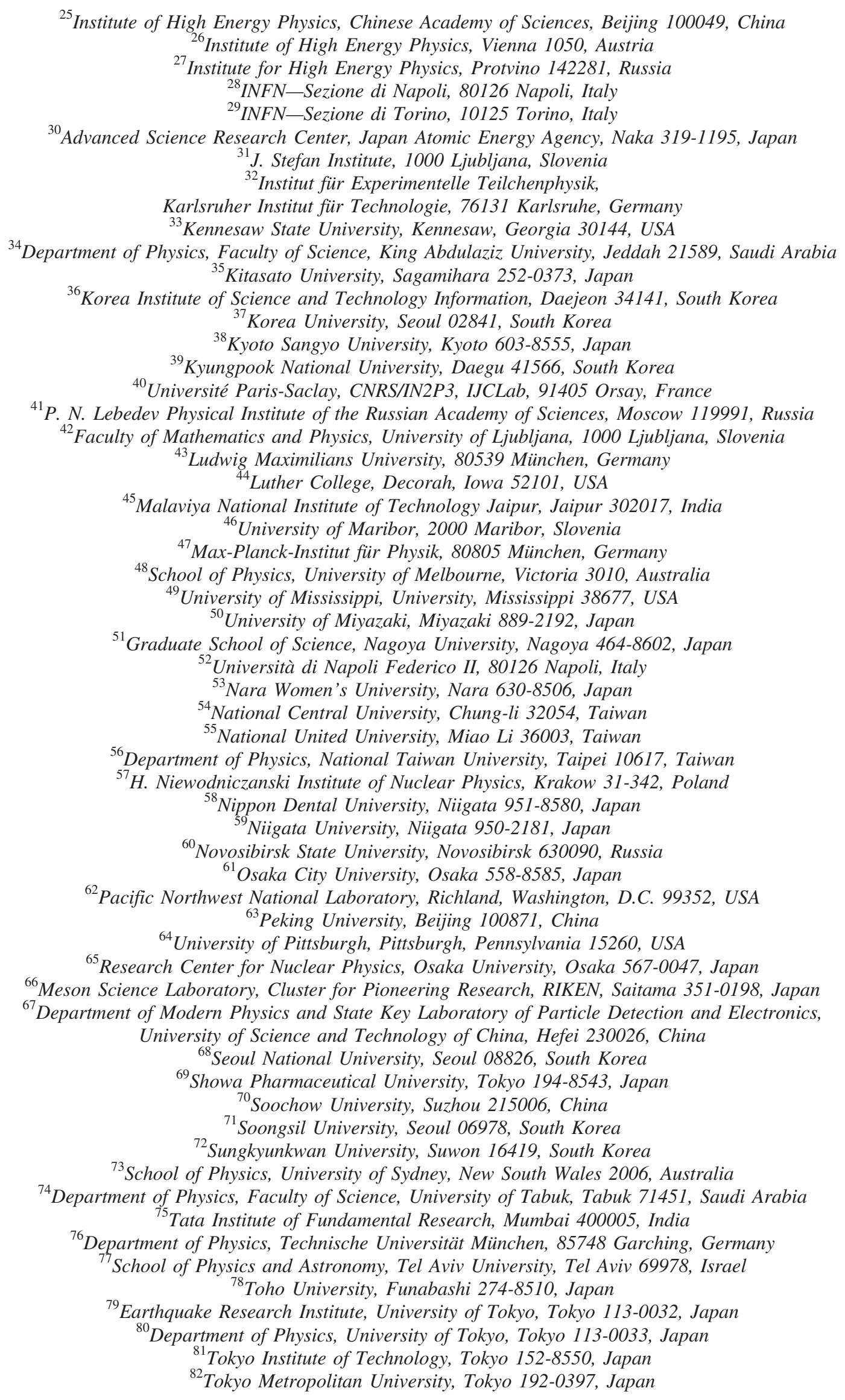




\author{
${ }^{83}$ Utkal University, Bhubaneswar 751004, India \\ ${ }^{84}$ Virginia Polytechnic Institute and State University, Blacksburg, Virginia 24061, USA \\ ${ }^{85}$ Wayne State University, Detroit, Michigan 48202, USA \\ ${ }^{86}$ Yamagata University, Yamagata 990-8560, Japan \\ ${ }^{87}$ Yonsei University, Seoul 03722, South Korea
}

(Received 11 December 2020; accepted 10 May 2021; published 3 June 2021)

\begin{abstract}
Using the entire data sample of $980 \mathrm{fb}^{-1}$ integrated luminosity collected with the Belle detector at the KEKB asymmetric-energy $e^{+} e^{-}$collider, we present an amplitude analysis measuring the branching fractions of the Cabibbo-allowed, $W$-exchange resonant decay $\Xi_{c}^{0} \rightarrow \Xi^{0} \phi\left(\rightarrow K^{+} K^{-}\right)$with a polarized $\phi$ and the nonresonant decay via a direct process $\Xi_{c}^{0} \rightarrow \Xi^{0} K^{+} K^{-}$. We present these measurements, relative to the normalization mode $\Xi^{-} \pi^{+}$, and find branching ratios $\frac{\mathcal{B}\left(\Xi_{c}^{0} \rightarrow \Xi^{0} \phi\left(\rightarrow K^{+} K^{-}\right)\right)}{\left(\mathcal{B} \Xi_{c}^{0} \rightarrow \Xi^{-} \pi^{+}\right)}=0.036 \pm 0.004$ (stat.) \pm 0.002 (syst.) and $\frac{\mathcal{B}\left(\Xi_{c}^{0} \rightarrow \Xi^{0} K^{+} K^{-}\right)}{\mathcal{B}\left(\Xi_{c}^{0} \rightarrow \Xi^{-} \pi^{+}\right)}=0.039 \pm 0.004$ (stat.) \pm 0.002 (syst.), which suggest that only minor cusping peaks occur in the combinatorial background of $\Omega^{*-} \rightarrow \Xi^{0} K^{-}$due to these $\Xi_{c}^{0}$ decays.

DOI: 10.1103/PhysRevD.103.112002
\end{abstract}

\section{INTRODUCTION}

The most simple model of the $\Xi_{c}^{0}(d s c)$ baryon nonleptonic decay occurs via $c \rightarrow s$ transitions into $\Xi^{-} \pi^{+}$by way of an emitted $W$ boson. However, these decays may also occur via charged-current ( $W$-exchange) interactions between the quarks of the $\Xi_{c}^{0}$ baryon. The most probable example of this decay is the Cabibbo-allowed $W$ exchange between the $c d$ quarks, as shown in the decay diagrams of Fig. 1. In this type of decay, the $c$ and $d$ quarks exchange charge via the interacting $W$ boson and transition into $s$ and $u$ quarks, respectively. Generally, the remaining momentum from this decay escapes via an emitted gluon, which immediately produces a quark-antiquark pair. For this work, we study the case in which the emitted gluon from the $W$ exchange decays into an $s \bar{s}$ quark pair ( $s \bar{s}$-popping) through $c d \rightarrow W^{+} \rightarrow s u(g \rightarrow s \bar{s})$.

Such $s \bar{s}$-popping decays are in general suppressed compared with the analogous light-quark-popping decays but have more cleanly measurable final states. Other $s \bar{s}$ popping decays have been measured, and in particular by the Belle Collaboration, the similar but Cabibbo-suppressed mode $\Xi_{c}^{0} \rightarrow \Lambda K^{+} K^{-}$[1]. In this work, we study the previously unobserved [2] Cabibbo-allowed mode $\Xi_{c}^{0} \rightarrow \Xi^{0} K^{+} K^{-}$and the fraction of this decay that resonates through $\phi \rightarrow K^{+} K^{-}$.

One particular motivation for the study of these new $\Xi_{c}^{0}$ decay channels is due to the recently discovered excited $\Omega^{-}$

\footnotetext{
*Now at Hiroshima University, Higashi-Hiroshima, Hiroshima 739-8530, Japan.

Published by the American Physical Society under the terms of the Creative Commons Attribution 4.0 International license. Further distribution of this work must maintain attribution to the author(s) and the published article's title, journal citation, and DOI. Funded by SCOAP ${ }^{3}$.
}

baryon at Belle [3]. This excited $\Omega^{-}$was found in the $\Xi^{0} K^{-}$ channel with an invariant mass of $2.012 \mathrm{GeV}$, where for this work we use natural units with $c=1$. From quark model predictions of heavy-baryon excited states, there are good theoretical reasons to expect that this $\Omega^{-}$baryon may have a partner near $1.95 \mathrm{GeV}[3,4]$, and low-statistics indications of an excess in this region can be inferred. However, it is suspected that a $\Xi_{c}^{0}$ decaying to $\Xi^{0} K^{+} K^{-}$through a polarized $\phi \rightarrow K^{+} K^{-}$could produce peaks in the $\Xi^{0} K^{ \pm}$ invariant mass spectra as well. These topological substructures are due to the helicity angles of the $\Xi_{c}^{0}$ polarizing the $\phi$ in the $1 / 2 \rightarrow 1 / 2+1$ resonant decay process [5]. Hence, the decay substructure of the $\Xi_{c}^{0} \rightarrow \Xi^{0} K^{+} K^{-}$must be studied to assure that any evidence of an excess in this

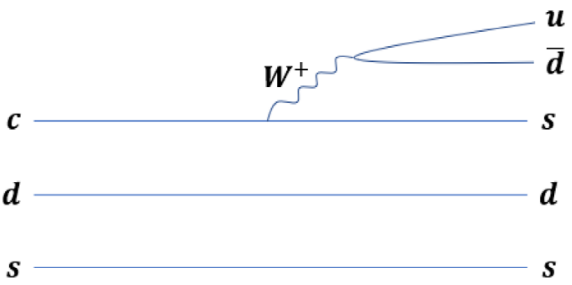

(a)

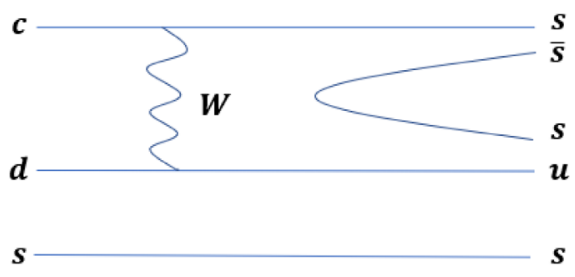

(b)

FIG. 1. Decay diagrams depicting (a) the nonleptonic, weak decay of $\Xi_{c}^{0} \rightarrow \Xi^{-} \pi^{+}$via an emitted $W$ boson and (b) the Cabibbo-allowed $W$-exchange, $s \bar{s}$-popping decay of $\Xi_{c}^{0} \rightarrow \Xi^{0} K^{+} K^{-}$, which can resonate through $\phi \rightarrow K^{+} K^{-}$. 
region can be attributed to an excited $\Omega^{-}$candidate and not an artifact of these resonant decays.

The Belle detector and KEKB asymmetric $e^{+} e^{-}$collider collected the world's largest sample of $\Upsilon(4 S)$ energy data over a 1999-2010 run period with a total $980 \mathrm{fb}^{-1}$ integrated luminosity for analysis across all energy ranges [6]. The Belle detector was a large, asymmetric detector hermetically consisting of six subdetectors. From inner- to outermost subdetector, the Belle detector included the following components.

For charged particle tracking, four innermost layers of double-sided silicon strip vertex detectors surrounded the beryllium beam pipe, encased by a 50-layer central drift chamber (CDC). These tracking detectors were asymmetrically oriented in the $z$ axis to assure optimal solid angle coverage with respect to the interaction point (IP). For charged particle identification (PID), defined $\mathcal{L}(x: y)=$ $\mathcal{L}_{x} /\left(\mathcal{L}_{x}+\mathcal{L}_{y}\right)$ for likelihoods $\mathcal{L}_{i}$ of identifying the charged particles $p, \pi$, and $K$, along with $d E / d x$ measurements in the CDC, Belle included two subdetectors. The aerogel Cherenkov counters (ACC) were located along the barrel and the outer, large solid angle of the CDC, and the time-offlight counters were positioned just outside of the ACC with respect to the barrel. For electron and $\gamma$ detection via electromagnetic showers, along the entire Belle detector $23^{\circ}<\theta<139^{\circ}$ solid angle, $\mathrm{CsI}(\mathrm{Tl})$ crystals comprised the electromagnetic calorimeter (ECL). Outside the $1.5 \mathrm{~T}$ solenoid coil, the remaining subdetectors included layered resistive plate counters with an iron yoke for muon and $K_{L}$ detection $[7,8]$.

\section{RECONSTRUCTION}

Event reconstruction for this analysis is performed entirely in the Belle II Software Framework by converting Belle data structures to Belle II data structures $[9,10]$. The background to the reconstructed invariant mass distribution of the $\Xi^{0} K^{+} K^{-}$signal is in large part due to combinatorics and clone hyperon reconstructions from soft and overlapping $\gamma$ candidates. Hence, it is necessary to reconstruct $\Xi^{0} \rightarrow \Lambda \pi^{0}$ candidates with a good signal-to-background ratio. We detail the reconstruction procedure and selection criteria for the $\Xi_{c}^{0}$ modes $-\Xi_{c}^{0} \rightarrow\left(\Xi^{-} \rightarrow\left(\Lambda \rightarrow p \pi^{-}\right) \pi^{-}\right) \pi^{+}$ and $\Xi_{c}^{0} \rightarrow\left(\Xi^{0} \rightarrow\left(\Lambda \rightarrow p \pi^{-}\right)\left(\pi^{0} \rightarrow \gamma \gamma\right)\right) K^{+} K^{-}$- by following a similar methodology to that of the $\Omega(2012) \rightarrow$ $\Xi^{0} K^{-}$observation [3], which used the previous Belle Software Framework.

Preliminary $\Lambda$ candidates must exhibit the expected kinematics from a long-lived hyperon decay, $\Xi \rightarrow \Lambda \pi$. These $\Lambda \rightarrow p \pi^{-}$candidates are found with a local vertex reconstruction using a Kalman filter [11] and selected with the following kinematics: a reconstructed mass in $\pm 3.5 \mathrm{MeV}$ range of the nominal mass [2] which is approximately $99 \%$ efficient, $\operatorname{a} \cos \left(\alpha_{x y z}\right)>0.0$ in the 3D plane, a distance of the decay vertex with respect to the IP greater than $0.35 \mathrm{~cm}$, and a loose PID requirement on the $p$ track with $\mathcal{L}(p: \pi)$ and $\mathcal{L}(p: K)$ greater than 0.2 which is approximately $99 \%$ efficient. For these kinematics, we define $\alpha_{x y z}\left(\alpha_{x y}\right)$ as the 3D (2D) angle between a vector from the IP to the decay vertex and the momentum vector at the decay vertex. All hyperons with higher strangeness are then reconstructed with the described $\Lambda$ candidates, and all good $\pi^{-}$or $\pi^{0} \rightarrow \gamma \gamma$ candidates are a priori selected by internal Belle studies of the CDC and ECL performance [10]. The decay vertex of these hyperon candidates is then globally reconstructed using a decay chain fitter with mass-constrained daughters to improve the signal-to-background ratio $[9,11]$.

Optimal $\Xi^{-} \rightarrow \Lambda \pi^{-}$selection is less crucial for the normalization mode decay due to the high yield of these charmed baryon decays produced at Belle. We loosely select $\Xi^{-} \rightarrow \Lambda \pi^{-}$candidates for this analysis with the following kinematics: a reconstructed invariant mass within a $\pm 3.5 \mathrm{MeV}$ range of the nominal mass [2], which is approximately $4 \sigma$ with respect to the primary resolution; a reconstructed decay vertex chi-squared probability consistent with a $\chi^{2}$ per degree of freedom less than 20; a distance of the $\Xi^{-}$decay vertex with respect to the IP preceding the $\Lambda$ decay vertex distance; a distance of the $\Xi^{-}$decay vertex with respect to the IP greater than $0.1 \mathrm{~cm}$; a $\pi^{-}$transverse momentum greater than $50 \mathrm{MeV}$; and a loose ratio between the $\Lambda$ and $\Xi^{-}$hyperon $\cos \left(\alpha_{x y}\right)$ angles in the tangential plane which is consistent with a hyperon decay. The reconstructed invariant mass for these $\Xi^{-} \rightarrow \Lambda \pi^{-}$hyperons is shown in Fig. 2 together with an unbinned maximum likelihood fit to the data using a probability density function (PDF) composed of a double Gaussian signal function and a second-order Chebyshev polynomial background.

We reconstruct $\Xi^{0} \rightarrow \Lambda \pi^{0}$ by refitting the $\pi^{0}$ due to the a priori candidates containing no directional information. Using a global decay chain reconstruction $[9,11]$, the $\pi^{0}$ candidates are refit to the decay vertex of the $\Xi^{0} \rightarrow \Lambda \pi^{0}$, with the $\Xi^{0}$ constrained to come from the nominal IP region. After refitting these $\pi^{0}$ candidates, the $\Xi^{0}$ hyperons used for this analysis are selected with the following kinematics: a reconstructed invariant mass within a $\pm 5 \mathrm{MeV}$ range of the nominal mass [2], which is approximately $2 \sigma$ with respect to the primary resolution; a reconstructed decay vertex chi-squared probability consistent with a $\chi^{2}$ per degree of freedom less than 20; a distance of the $\Xi^{0}$ decay vertex with respect to the IP preceding the $\Lambda$ decay vertex distance; a distance of the $\Xi^{0}$ decay vertex with respect to the IP greater than $1.4 \mathrm{~cm}$; a refit $\pi^{0}$ momentum greater than $150 \mathrm{MeV}$; a refit $\pi^{0}$ mass range $\pm 10.4 \mathrm{MeV}$ about the $\pi^{0}$ nominal mass [2], which is approximately $2 \sigma$; and lastly, a $\Lambda$ tangential $\alpha_{x y}$ deflection angle greater than the IP-produced $\Xi^{0}$ angle of $\alpha_{x y} \approx 0$. The reconstructed invariant mass for these $\Xi^{0} \rightarrow \Lambda \pi^{0}$ hyperons is shown in Fig. 2, together with an unbinned maximum- 

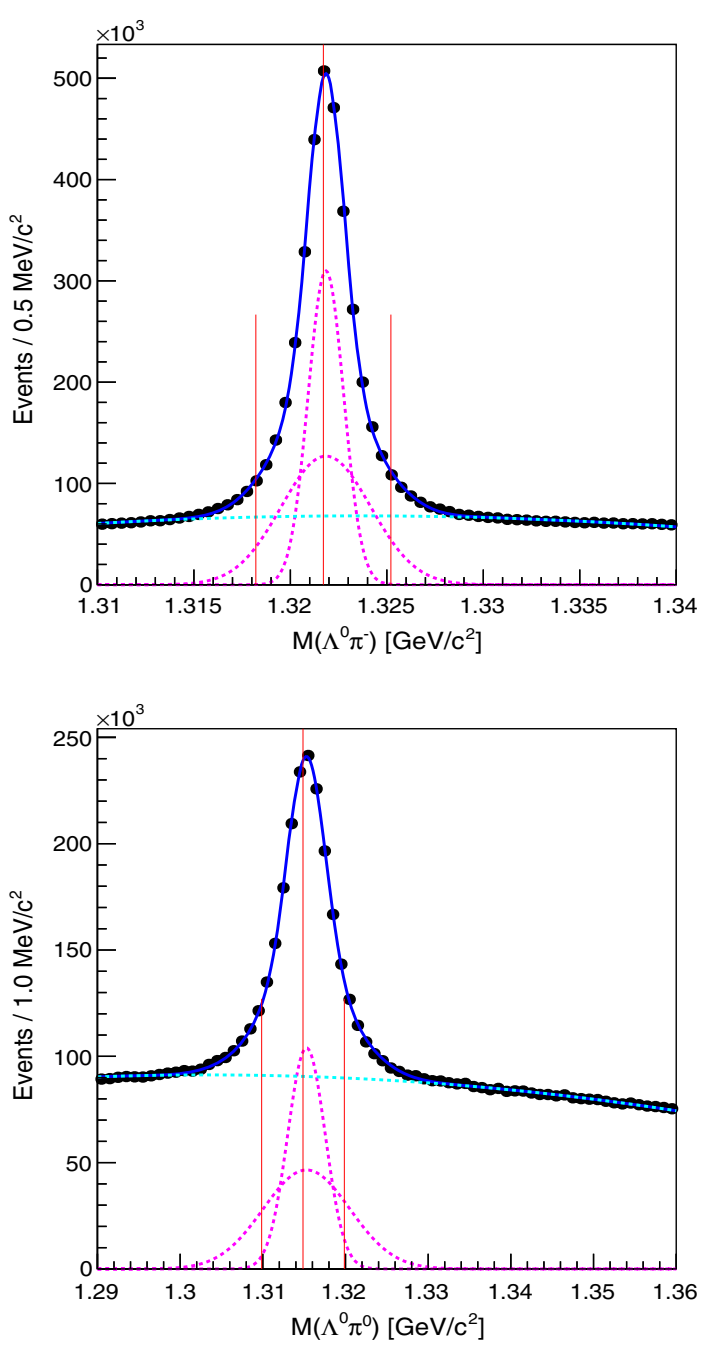

FIG. 2. Selected signal bands (marked in red half lines) with respect to the nominal masses (marked in red full lines) of the invariant mass distributions for the hyperon decays $\Xi^{-} \rightarrow \Lambda \pi^{-}$ (upper) and $\Xi^{0} \rightarrow \Lambda \pi^{0}$ (lower) in the data sample (black) with an unbinned likelihood fit to a double Gaussian PDF (solid blue) with a Chebyshev polynomial background (dashed cyan) and each individual Gaussian contribution to the signal PDF (dashed magenta).

likelihood fit to the data using a PDF composed of a double Gaussian signal function and a second-order Chebyshev polynomial background.

For the normalization channel $\Xi_{c}^{0} \rightarrow \Xi^{-} \pi^{+}$, all $\Xi^{-}$ hyperons described above are mass-constrained and combined with selected $\pi^{+}$candidates. These $\pi^{+}$candidates are selected with a point of closest approach (POCA) in the $x y$ plane less than $0.2 \mathrm{~cm}$, a POCA along the $z$ axis less than $1.0 \mathrm{~cm}$, and a loose PID requirement with $\mathcal{L}(\pi: p)$ and $\mathcal{L}(\pi: K)$ greater than 0.2 , which is approximately $99 \%$ efficient. To reconstruct the signal channel $\Xi_{c}^{0} \rightarrow \Xi^{0} K^{+} K^{-}$, the $\Xi^{0}$ hyperons described above are mass-constrained and combined with similarly selected $K^{ \pm}$candidates. These $K^{ \pm}$ candidates are selected with a POCA in the $x y$ plane less than $0.2 \mathrm{~cm}$, a POCA along the $z$ axis less than $1.0 \mathrm{~cm}$, and tight PID requirements, with $\mathcal{L}(K: p)$ and $\mathcal{L}(K: \pi)$ greater than 0.9 , which is approximately $83 \%$ efficient. The decay vertex of these $\Xi_{c}^{0}$ candidates in each mode is then reconstructed locally and constrained to the nominal IP profile region of the Belle detector. The final $\Xi_{c}^{0}$ candidates are then optimally selected via a figure of merit with a scaled momentum $x_{p}>0.5$ for $x_{p}=p^{*} / \sqrt{E_{\text {beam }}^{2}-M_{\Xi_{c}^{0}}^{2}}$, where $p^{*}$ is the momentum in the $e^{+} e^{-}$center-of-mass (c.m.) frame. This requirement is typically used to produce a good signal-to-background ratio while retaining high efficiency for charmed baryons produced in $e^{+} e^{-} \rightarrow q \bar{q}$ continuum events.

From the Monte Carlo (MC), the PDF which best describes the $\Xi_{c}^{0}$ baryon is a double Gaussian with a primary, core, resolution, and secondary resolution along with a second-order Chebyshev polynomial background. In Fig. 3, we plot the results of these unbinned maximumlikelihood fits to the invariant mass distributions of the normalization channel, $\Xi_{c}^{0} \rightarrow \Xi^{-} \pi^{+}$, and the signal channel, $\Xi_{c}^{0} \rightarrow \Xi^{0} K^{+} K^{-}$, with fixed primary and secondary resolutions from MC simulations but with a free parameter yield into each Gaussian. We define the reconstruction efficiency into each mode as a ratio of signal yields between the reconstruction level and generator level for generic MC events containing a single generated $\Xi_{c}^{0}$.

For the normalization mode, the primary and secondary resolutions are independently found to be $7.1 \pm 0.1 \mathrm{MeV}$ and $14.5 \pm 0.2 \mathrm{MeV}$, respectively, which correspond to a rms resolution of $9.7 \pm 0.1 \mathrm{MeV}$ and has a reconstruction efficiency $\epsilon_{\Xi^{-} \pi^{+}}=(7.04 \pm 0.05) \%$ with respect to the generated MC when resolutions are constrained. For the nonresonant and resonant signal modes, these resolutions are determined independently, from a $\Xi_{c}^{0} \rightarrow \Xi^{0} K^{+} K^{-} \mathrm{MC}$ generated with phase space distribution and a $\Xi_{c}^{0} \rightarrow \Xi^{0} \phi(\rightarrow$ $K^{+} K^{-}$) MC generated with a helicity amplitude distribution $[5,12,13]$, respectively. The difference in resolution between the resonant and nonresonant decays was found to be negligible, and they are averaged with equal weighting. These average primary and secondary resolutions are found to be $3.19 \pm 0.02 \mathrm{MeV}$ and $12.37 \pm 0.21 \mathrm{MeV}$, respectively, and correspond to a rms resolution of $5.6 \pm 0.1 \mathrm{MeV}$. Each signal-mode MC sample, $\Xi_{c}^{0} \rightarrow$ $\Xi^{0} K^{+} K^{-}$and $\Xi_{c}^{0} \rightarrow \Xi^{0} \phi\left(\rightarrow K^{+} K^{-}\right)$, has statistically equivalent reconstruction efficiencies for its own resolution constraints, given as $\epsilon_{\Xi^{0} K^{+} K^{-}}=(1.08 \pm 0.01) \%$ and $\epsilon_{\Xi^{0} \phi}=$ $(1.09 \pm 0.01) \%$, respectively, within a $\pm 10 \mathrm{MeV}$ range of the $\Xi_{c}^{0}$ nominal mass [2], which is approximately $3 \sigma$ with respect to the primary resolution.

From sideband samples in the $\Xi^{0} K^{+} K^{-}$invariant mass distribution, we find that a significant number of $\phi \rightarrow$ $K^{+} K^{-}$decays which are not from a resonant $\Xi_{c}^{0}$ are reconstructed in the $\pm 10 \mathrm{MeV}$ signal band. To account for these events, as well as the uniform combinatorial 

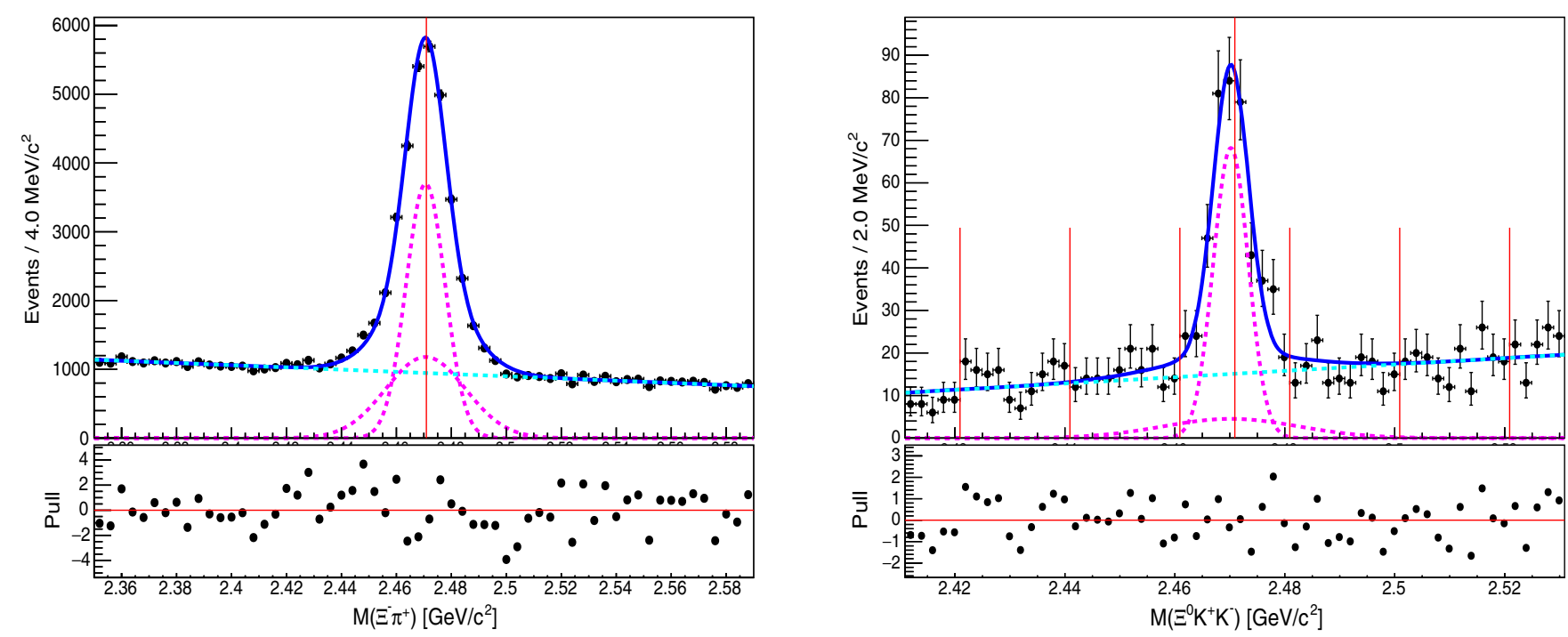

FIG. 3. Reconstructed invariant mass distributions for the normalization mode $\Xi_{c}^{0} \rightarrow \Xi^{-} \pi^{+}$(left) and the analysis mode $\Xi_{c}^{0} \rightarrow$ $\Xi^{0} K^{+} K^{-}$(right) in the data sample (black) with an unbinned likelihood fit to a double Gaussian PDF (solid blue) using fixed MC resolutions with a Chebyshev polynomial background (dashed cyan) and each individual Gaussian contribution to the signal PDF (dashed magenta). The pull distribution for each likelihood fit is included (bottom) adjacent to each reconstructed distribution. The selected signal, left, and right sidebands (marked in red half lines) with respect to the nominal masses (marked in red full lines) are described in the text.

background, all reconstructed signal candidates in the signal band range of the $\Xi_{c}^{0}$ nominal mass [2] are sideband-subtracted. This is done by subtraction of the scaled sideband candidates in the left and right sidebands of equal $\pm 10 \mathrm{MeV}$ width at central mass energies (labeled in Fig. 3) of $\pm 40 \mathrm{MeV}$ to the nominal mass with respect to the $\pm 10 \mathrm{MeV}$ signal band.

From the resulting likelihood fits in Fig. 3 with resolutions constrained to the MC values previously defined,

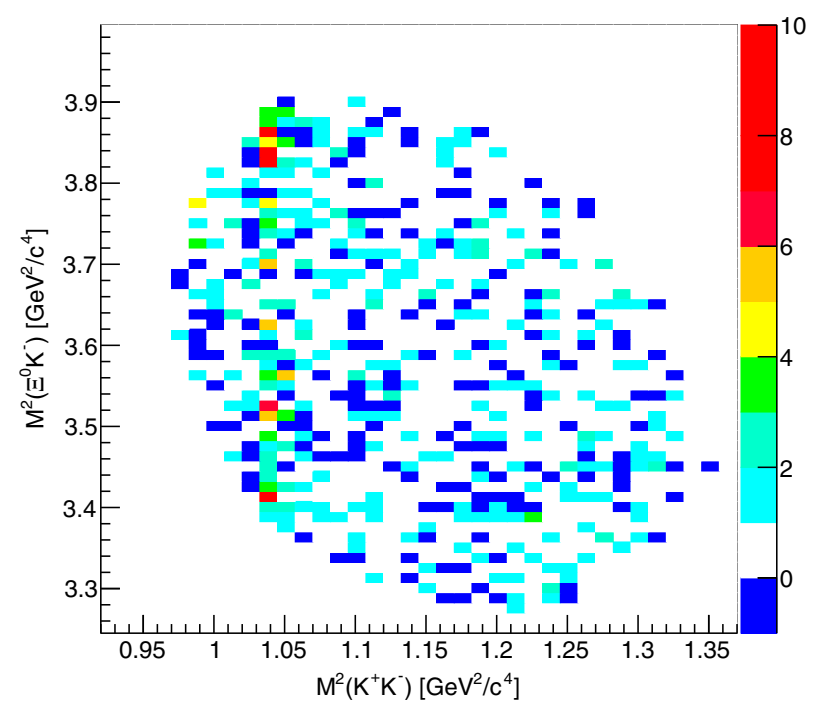

FIG. 4. Dalitz plot distribution of the $\Xi_{c}^{0} \rightarrow \Xi^{0} K^{+} K^{-}$decays in the sideband-subtracted $\Xi_{c}^{0}$ signal region of the data sample, shown with square bins of $12.5 \mathrm{MeV}$ width. we find $n_{\Xi^{+} \pi^{-}}=27186 \pm 475$ normalization-mode candidates over the entire distribution range. Similarly, within the $\pm 10 \mathrm{MeV}$ range of the nominal mass, we find $n_{\text {cand. }}=$ $n_{\Xi^{0} \phi}+n_{\Xi^{0} K^{+} K^{-}}=311 \pm 23$ candidates in both signal modes by using a scaled sum of events in the sidebandsubtracted distribution of the $\Xi^{0} K^{+} K^{-}$invariant mass channel. Defining a signal's statistical significance as $s / \delta(s)$ for signal yield $s$ and its uncertainty $\delta(s)$, our results correspond to a $13.5 \sigma$ statistical significance of these new Cabibbo-allowed $W$-exchange decays of $\Xi_{c}^{0} \rightarrow$ $\Xi^{0} K^{+} K^{-}$including the resonant mode via $\phi \rightarrow K^{+} K^{-}$.

The sideband-subtracted Dalitz plot is shown in Fig. 4 with a mass-constrained $\Xi_{c}^{0}$ final state across the entire phase space. From this figure, we find a clear $\phi \rightarrow K^{+} K^{-}$ band, but no evidence of other resonances. The $\phi$ resonance is found to be nonuniform due to the spin polarization of the $\phi$ in the $1 / 2 \rightarrow 1 / 2+1$ resonant decay process $\Xi_{c}^{0} \rightarrow \Xi^{0} \phi$. This nonuniform substructure is specifically observed near $M^{2}\left(\Xi^{0} K^{-}\right) \approx 3.85 \mathrm{GeV}^{2}$ and $M^{2}\left(\Xi^{0} K^{-}\right) \approx$ $3.425 \mathrm{GeV}^{2}$ along the $\phi$ band at $M^{2}\left(K^{+} K^{-}\right) \approx 1.04 \mathrm{GeV}^{2}$. For this work, we define the branching ratios $\frac{\mathcal{B} \text { (mode) }}{\mathcal{B}\left(\Xi_{c}^{0} \rightarrow \Xi^{-} \pi^{+}\right)}=$ $\frac{n_{\text {mode }}}{\epsilon_{\text {mode }}} / \frac{n_{\Xi^{-} \pi^{+}}}{\epsilon_{\Xi^{-}} \pi^{+}}$with previously defined efficiencies. Using this definition, we study the resonant and non-resonant fractions of $\Xi_{c}^{0} \rightarrow \Xi^{0} K^{+} K^{-}$using an amplitude analysis over the entire phase space of this decay.

\section{AMPLITUDE MODEL}

The nonuniform contributions to the resonant substructure in the $\Xi_{c}^{0} \rightarrow \Xi^{0} \phi$ decay are best modeled using an 


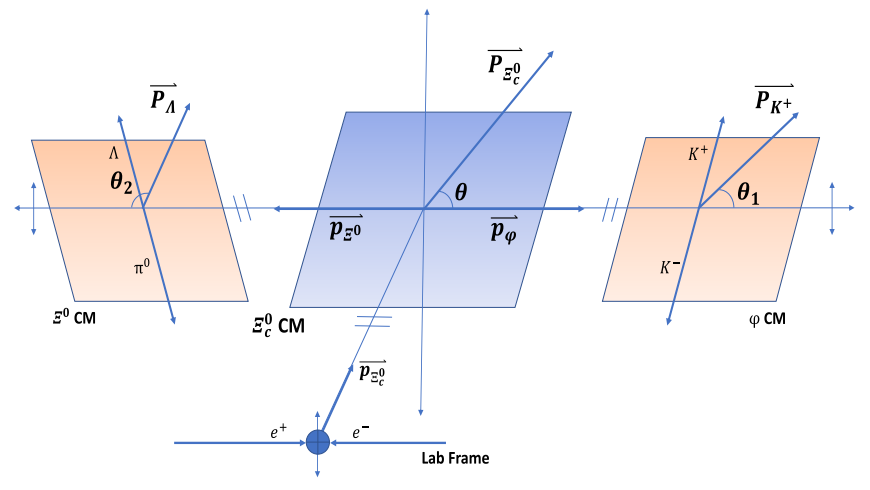

FIG. 5. Azimuthally symmetric diagram of the spin-polarization angles in the resonant $\Xi_{c}^{0} \rightarrow \Xi^{0} \phi\left(\rightarrow K^{+} K^{-}\right)$decay.

amplitude analysis over the decay phase space. In this section, we provide basic forms used to model the spinpolarized, resonant amplitudes for $\phi \rightarrow K^{+} K^{-}$from the spin-1/2 $\Xi_{c}^{0}$ in an azimuthally symmetric plane. From the branching fractions into the resonant and nonresonant modes in Fig. 4 and the efficiency-corrected integration of amplitude intensities [14], we calculate the branching ratios $\frac{\mathcal{B}\left(\Xi_{c}^{0} \rightarrow \Xi^{0} \phi\left(\rightarrow K^{+} K^{-}\right)\right)}{\mathcal{B}\left(\Xi_{c}^{0} \rightarrow \Xi^{-} \pi^{+}\right)}$and $\frac{\mathcal{B}\left(\Xi_{c}^{0} \rightarrow \Xi^{0} K^{+} K^{-}\right)}{\mathcal{B}\left(\Xi_{c}^{0} \rightarrow \Xi^{-} \pi^{+}\right)}$as previously defined for the amplitude analysis.

It is known that a resonant decay amplitude is well modeled to first order using a Breit-Wigner amplitude $[1,8]$. To higher orders, nonisotropic angular distributions contribute to the resonant amplitude due to the spin orientations of the final-state decay products. For the resonant $\Xi_{c}^{0} \rightarrow \Xi^{0} \phi\left(\rightarrow K^{+} K^{-}\right)$decay, the unit spin of the $\phi$ meson is polarized due to the $1 / 2$ spin of the heavy baryons. To describe this angular dependence of the polarized $\phi$, we study the polar polarization angles for the resonant $\Xi_{c}^{0}$ decay shown in Fig. 5 with respect to the $\Xi_{c}^{0}$ momentum in the lab frame. In contrast, we assume the simple model that nonresonant $\Xi_{c}^{0} \rightarrow \Xi^{0} K^{+} K^{-}$are uniform and isotropic, and are modeled using a constant amplitude over the entire phase space.

From Fig. 5, $\Xi_{c}^{0}$ decays resonantly into a $\phi$ with a polarization angle $\theta$ with respect to its polarization vector $\overrightarrow{P_{\Xi_{c}^{0}}}$ in the lab frame. The polarization angle $\theta$ in this diagram is defined as the angle between the polarization vector and the resonant daughter momentum vector, $\overrightarrow{P_{\phi}}$, in the $\Xi_{c}^{0}$ c.m. frame. Similarly, the resonant, spin- $1 \phi$ and spin-1/2 $\Xi^{0}$ decay with corresponding polarization angles $\theta_{1}$ and $\theta_{2}$ with respect to their momenta in the $\Xi_{c}^{0} \mathrm{c} . \mathrm{m}$. frame and their daughter polarization vectors, $\overrightarrow{P_{\Lambda}}$ and $\overrightarrow{P_{K^{+}}}$, in their own respective c.m. frame.

After integration over $\theta_{2}$ in the Dalitz plot, the angular distribution of the resonant $\Xi_{c}^{0}$ decay depends effectively on the polarization angles of the $\Xi_{c}^{0}$ and the resonant $\phi$ coupled by their corresponding helicity amplitudes, $H_{\lambda_{\phi}, \lambda_{\Xi^{0}}}$. We define this effective angular distribution of the resonant $\Xi_{c}^{0}$ decay amplitude as $A\left(\theta, \theta_{1}\right) \propto$ $\mathbf{d}_{\lambda_{\phi}, \lambda_{K^{+}}-\lambda_{K^{-}}}^{1}\left(\theta_{1}\right) H_{\lambda_{\phi}, \lambda_{\Xi^{0}}} \mathbf{d}_{\lambda_{\Xi_{c}}, \lambda_{\phi^{-}}-\lambda_{\Xi^{0}}}^{\mathbf{1} / \mathbf{2}}(\theta)$ for small Wigner Dfunctions $\mathbf{d}_{\lambda, \lambda_{1}-\lambda_{2}}^{\mathbf{j}}$ of a parent particle with spin $\mathbf{j}$ and helicity $\lambda$ decaying into two daughters with helicities $\lambda_{1}$ and $\lambda_{2}[5,12]$. The kinematics in this effective angular distribution of our amplitude model are given in Fig. 6 for $\cos (\theta)$ and $\cos \left(\theta_{1}\right)$ within a $\pm 20 \mathrm{MeV}$ range of the resonant nominal mass and in nonresonant regions of phase space.

When fitting the Dalitz plot using this angular distribution, $A\left(\theta, \theta_{1}\right)$, due to the low statistics, we reparametrize the sum of helicity contributions by absorbing constants into the overall normalization and eliminate any direct $H_{\lambda_{\phi}, \lambda_{\Sigma^{0}}}$ dependence, which is outside the scope of this work. The resulting ratios of $H_{\lambda_{\phi}, \lambda_{\Xi^{0}}}$ amplitudes are then free parameters in the amplitude model. Among these ratios, only the
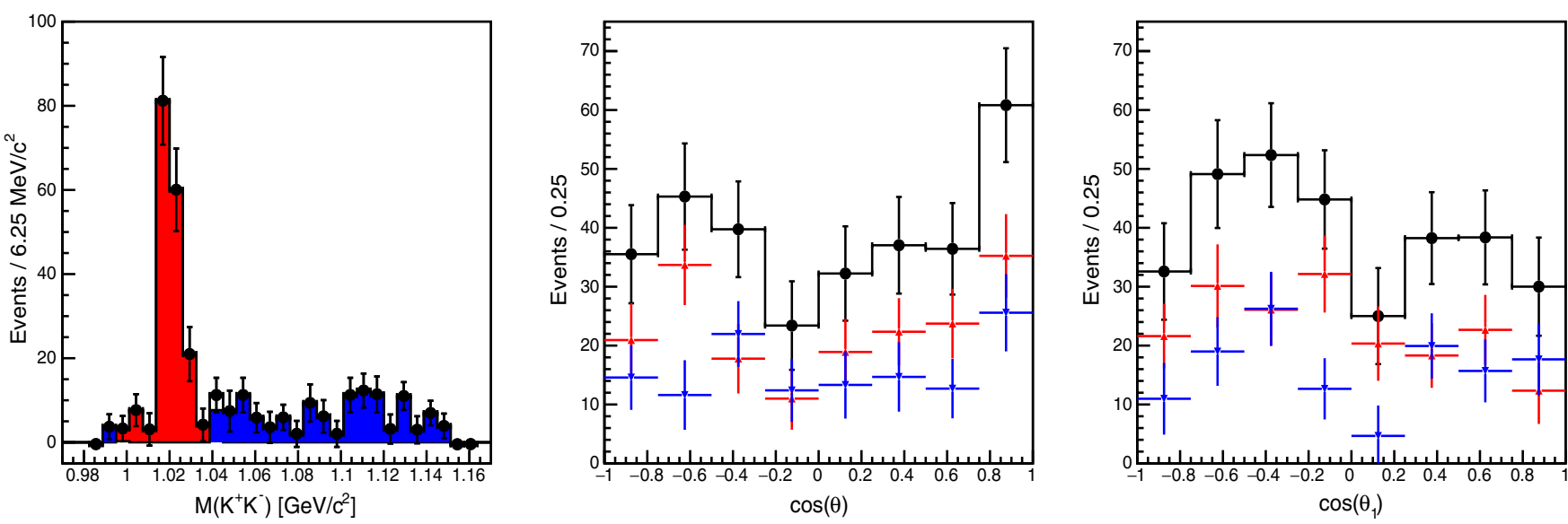

FIG. 6. Angular kinematics in the sideband-subtracted data sample for the $\cos (\theta)$ (middle) and $\cos \left(\theta_{1}\right)$ (right) distributions (black) within a $\pm 20 \mathrm{MeV}$ range of the resonant nominal mass (red) and in nonresonant regions of phase space (blue) selected about the $K^{+} K^{-}$ invariant mass projection (left). 
$\lambda_{\phi}=0,1$ terms contribute to the Dalitz plot, which corresponds to the integrated distribution over both $\theta$ and $\theta_{2}$ and will only depend on $\theta_{1}$ directly.

From this, we conclude that the $\Xi_{c}^{0} \rightarrow \Xi^{0} K^{+} K^{-}$amplitude from resonant, polarized $\phi \rightarrow K^{+} K^{-}$decays can be described by combining this effective angular distribution, $A\left(\theta, \theta_{1}\right)$, with a Breit-Wigner amplitude, $V\left(E, M_{\phi}, \Gamma\right)$. We then assert that this resonant amplitude is complex and coherent with the nonresonant constant amplitude, $\mathcal{A}_{K K}$, across the Dalitz plot distribution described as $\mid \mathcal{A}_{K K}+$ $\left.\mathcal{A}_{\phi} \sum V\left(E, M_{\phi}, \Gamma\right) A\left(\theta, \theta_{1}\right)\right|^{2}$ summed over all helicity states of $\Xi_{c}^{0}, \Xi^{0}$, and $\phi$ for normalization amplitudes $\mathcal{A}_{K K}$ and $\mathcal{A}_{\phi}$ of the nonresonant and resonant decays, respectively. Alternate hypotheses for these assumptions are included in the systematic uncertainties.

\section{AMPLITUDE ANALYSIS}

We fit the Dalitz plot distribution in Fig. 4 as a coherent sum of resonant and nonresonant amplitudes outlined in Sec. III. We then freely vary the helicity amplitude ratios of the resonant decay in the reparametrized angular distribution using only the natural width of the $\phi$ resonance, $\Gamma=$ 4.249 MeV [2], as a constraint. The convolution of this natural width with the Gaussian resolution is included in the systematic uncertainties. The result of this unbinned maximum-likelihood fit is shown in Fig. 7 across each pair of invariant mass projections using the amplitude analysis software AmpTools (v.10.2) [14]. From the normalized integration of each amplitude relative to the integral of their coherent sum, we measure branching fractions into the resonant and nonresonant modes as $(48.1 \pm 4.2) \%$ and $(51.9 \pm 4.2) \%$, respectively. In addition, we find that the measured mass of the $\phi$ meson, $M_{\phi}=1019.62 \pm$ $0.25 \mathrm{MeV}$, is in agreement with the current average value [2].
These branching fractions for the resonant and nonresonant decay modes correspond to $n_{\Xi^{0} \phi}=149 \pm 17$ and $n_{\Xi^{0} K^{+} K^{-}}=161 \pm 18$ candidates, respectively. After varying all free parameters of our amplitude model [14] and considering the reconstruction efficiencies across the Dalitz plot distribution from signal MC, we measure the precise resonant and nonresonant branching ratios into these new $W$-exchange $s \bar{s}$-popping decay modes:

$$
\begin{gathered}
\frac{\mathcal{B}\left(\Xi_{c}^{0} \rightarrow \Xi^{0} \phi\left(\rightarrow K^{+} K^{-}\right)\right)}{\mathcal{B}\left(\Xi_{c}^{0} \rightarrow \Xi^{-} \pi^{+}\right)}=0.036 \pm 0.004 \text { (stat.) } \\
\frac{\mathcal{B}\left(\Xi_{c}^{0} \rightarrow \Xi^{0} K^{+} K^{-}\right)}{\mathcal{B}\left(\Xi_{c}^{0} \rightarrow \Xi^{-} \pi^{+}\right)}=0.039 \pm 0.004 \text { (stat.) }
\end{gathered}
$$

The branching ratios in Eqs. (1) and (2) can easily be verified by one-dimensional analysis methods on the $\Xi^{0} K^{+} K^{-}$invariant mass distribution in Fig. 3. For this validation, we plot the invariant mass of the $K^{+} K^{-}$under the signal $\Xi_{c}^{0}$ band and weight the left- and right-sideband events appropriately, akin to the amplitude analysis. We then model the resonant $\phi \rightarrow K^{+} K^{-}$in the $K^{+} K^{-}$projection with a P-wave relativistic Breit-Wigner PDF convolved with a double Gaussian using constrained resolutions from signal MC studies. Using this analysis method, we find statistically consistent results corresponding to branching fractions $(48.7 \pm 4.9) \%$ and $(51.3 \pm 4.9) \%$ into the resonant and nonresonant modes, respectively. The corresponding minute differences between these measurements as branching ratios are consistent with a slight overestimation of the resonant mode observed during the $\mathrm{MC}$ analysis of this channel using one-dimensional methods. The methodological systematic uncertainty between the amplitudeanalysis and the one-dimensional-analysis branching ratios is included in the total systematic uncertainty of the final branching ratio measurement.
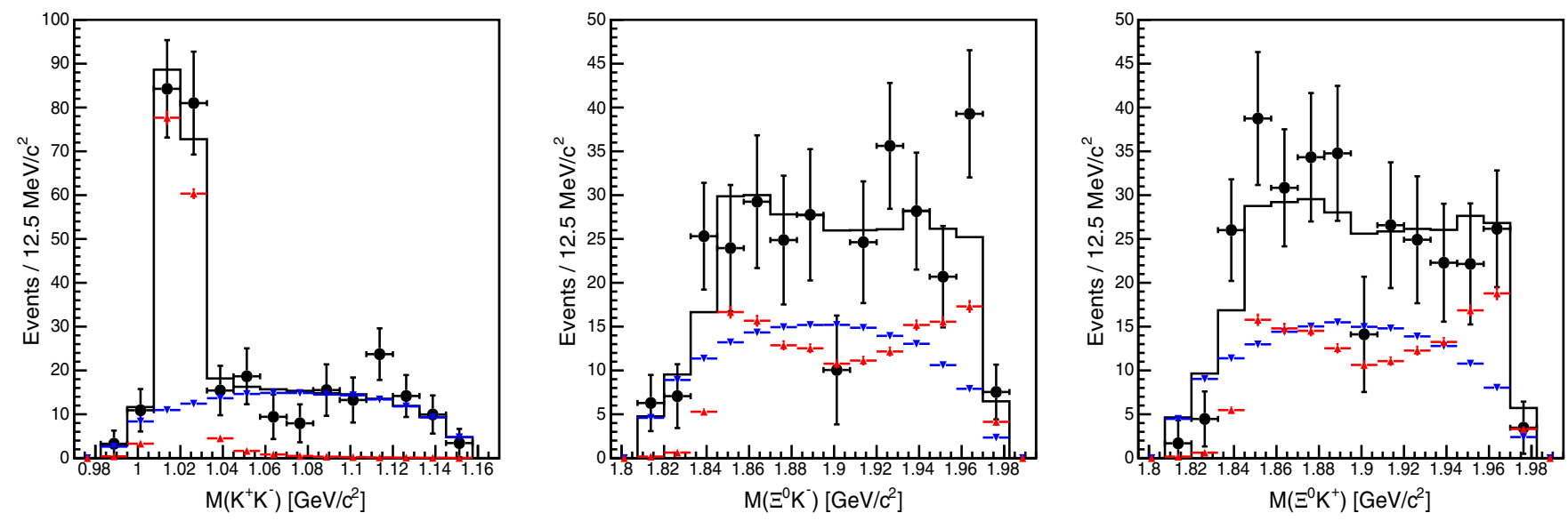

FIG. 7. Paired invariant mass projections of the amplitude intensities in the $\Xi_{c}^{0} \rightarrow \Xi^{0} K^{+} K^{-}$decay via the resonant $\phi \rightarrow K^{+} K^{-}$(red) and nonresonant (blue) modes in the sideband-subtracted data sample (black plot points) using the coherent sum of azimuthally symmetric amplitudes (solid black) as a model. 


\section{SYSTEMATIC UNCERTAINTIES}

In Table I, we summarize all systematic uncertainties which impact our branching ratio measurements, summed in quadrature, to calculate the total systematic uncertainty. As the branching fraction is defined by the ratio of $\Xi_{c}^{0} \rightarrow$ $\left(\Xi^{0} \rightarrow \Lambda^{0} \pi^{0}\right) K^{+} K^{-}$and $\Xi_{c}^{0} \rightarrow\left(\Xi^{-} \rightarrow \Lambda^{0} \pi^{-}\right) \pi^{+}$, the detection-efficiency-relevant (DER) errors from particle identification and reconstruction common to both modes, such as $\Lambda$ finding and tracking, cancel. The remaining uncertainties are due to $\pi^{0}$ reconstruction $( \pm 1.5 \%)$ and PID $( \pm 1.3 \%)$, giving a total $\pm 2.0 \%$ when quadratically summed. These are estimated by comparing data and MC for dedicated calibration samples. In addition, we assign a systematic uncertainty of ${ }_{-1.6}^{+0.0} \%$ and ${ }_{-3.3}^{+0.0} \%$ to each respective branching ratio measurement due to the DER errors from multiple candidates in the signal distributions as a result of cloned hyperon reconstructions and tracks which originate far from the IP. This is calculated by comparing measurements from MC samples to the truth generated.

In order to check our methodology, we compare the results found for the branching ratios with the amplitude analysis to those found using the simpler one-dimensional approach of fitting the Dalitz plot projections. We assign a systematic uncertainty of ${ }_{-0.0}^{+1.0} \%$ and ${ }_{-0.9}^{+0.0} \%$ to each respective branching ratio measurement due to variances between this amplitude analysis and the one-dimensional analysis measurements.

Our remaining systematic uncertainties are due to the MC statistics assumed for the resolutions as well as all model assumptions used throughout this analysis. We assign a collective systematic uncertainty of ${ }_{-0.1}^{+0.5} \%$ to each branching ratio measurement due to the $\mathrm{MC}$ assumptions in our PDF models. This is calculated by varying and floating the values of the resolution constraints about the statistical uncertainty and summing these effects in quadrature. Similarly, we assign a systematic uncertainty of ${ }_{-0.0}^{+0.8} \%$ to each branching ratio measurement due to the choice of PDF models used in our measurements of the $\Xi_{c}^{0}$ modes. This is calculated by finding the change in the result if we use a Gaussian PDF or a reduced fit range when modeling the reconstructed $\Xi_{c}^{0}$ invariant masses.

For the amplitude model assumptions, we assign a collective systematic uncertainty of ${ }_{-4.0}^{+3.8} \%$ and ${ }_{-3.8}^{+3.3} \%$ to each respective branching ratio measurement when quadratically summed. These are calculated by varying the model assumptions correlating to the natural width constraint [2] and various measurements with alternative or ancillary amplitude models. These collective calculations include the following systematic effects: the quality of the phase-space efficiencies and generated MC, which is calculated by measuring these branching ratios with integrated efficiencies $\left({ }_{-0.3}^{+0.0} \%\right.$ and $\left.{ }_{-0.0}^{+0.3} \%\right)$; the effect of smearing the Breit-Wigner natural width by the reconstruction resolution $\left({ }_{-4.0}^{+2.9} \%\right.$ and $\left.{ }_{-2.5}^{+3.2} \%\right)$; the effect of using incoherent, noninterference models of the two amplitudes $(< \pm 0.1 \%)$; the effect of including contributions due to $a_{0}(980)$ and $f_{0}(980)$ mesons near threshold $(< \pm 0.1 \%)$; the assumption of azimuthal symmetry found by perturbing the model about small Euler angles $( \pm 0.1 \%)$; and the effect of direct helicity amplitude dependencies, the reparametrization, and the defined polarization angles calculated by systematically varying and eliminating these free parameters in our amplitude model $\left({ }_{-0.5}^{+2.3} \%\right.$ and $\left.{ }_{-2.8}^{+0.4} \%\right)$.

Summing all of these systematic uncertainties in quadrature, as shown in Table I, we assign a total systematic

TABLE I. Contributions to the total systematic uncertainty of the branching ratio measurements, expressed as a percentage.

\begin{tabular}{lcc}
\hline \hline & Systematic uncertainties & \\
\hline & $\frac{\mathcal{B}\left(\Xi_{c}^{0} \rightarrow \Xi^{0} \phi\left(\rightarrow K^{+} K^{-}\right)\right)}{\mathcal{B}\left(\Xi_{c}^{0} \rightarrow \Xi^{-} \pi^{+}\right)}$ & $\frac{\mathcal{B}\left(\Xi_{c}^{0} \rightarrow \Xi^{0} K^{+} K^{-}\right)}{\mathcal{B}\left(\Xi_{c}^{0} \rightarrow \Xi^{-} \pi^{+}\right)}$ \\
\hline & Reconstruction & \\
$\pi^{0} \rightarrow \gamma \gamma$ \& PID & \pm 2.0 & +2.0 \\
& Multiple candidates & \\
Clones \& tracking & +0.0 & +0.0 \\
& One-dimensional analysis & -3.3 \\
$M\left(K^{+} K^{-}\right)$yield & -1.0 & +0.0 \\
& MC $\&$ model assumptions & -0.9 \\
MC resolutions & +0.5 & +0.5 \\
PDF Models & -0.1 & -0.1 \\
Amplitude models & +0.8 & +0.8 \\
& -0.0 & -0.0 \\
& +3.8 & +3.3 \\
& -4.0 & -3.8 \\
\hline \hline
\end{tabular}


certainty of ${ }_{-4.8}^{+4.5} \%$ and ${ }_{-5.5}^{+4.0} \%$ for the two branching ratio measurements, respectively.

\section{CONCLUSIONS}

Using the entire data sample of $980 \mathrm{fb}^{-1}$ integrated luminosity collected with the Belle detector [6], we find a signal with a statistical significance of $13.5 \sigma$ for new $W$ exchange $s \bar{s}$-popping decay modes of $\Xi_{c}^{0} \rightarrow \Xi^{0} K^{+} K^{-}$ including resonant decays through $\phi \rightarrow K^{+} K^{-}$. Using an azimuthally symmetric amplitude model, we find that among the $311 \pm 23$ candidates, $(48.1 \pm 4.2) \%$ decay resonantly through $\phi \rightarrow K^{+} K^{-}$, while $(51.9 \pm 4.2) \%$ decay directly to $\Xi^{0} K^{+} K^{-}$. These yields are directly compared to the normalization mode $\Xi_{c}^{0} \rightarrow \Xi^{-} \pi^{+}$of yield $27186 \pm 475$ over the same data sample. From these measurements and the previously studied reconstruction efficiencies on signal MC, we report new branching ratios for these resonant and nonresonant $\Xi_{c}^{0}$ modes:

$$
\begin{aligned}
& \frac{\mathcal{B}\left(\Xi_{c}^{0} \rightarrow \Xi^{0} \phi\left(\rightarrow K^{+} K^{-}\right)\right)}{\mathcal{B}\left(\Xi_{c}^{0} \rightarrow \Xi^{-} \pi^{+}\right)} \\
& \quad=0.036 \pm 0.004 \text { (stat.) } \pm 0.002 \text { (syst.) } \\
& \frac{\mathcal{B}\left(\Xi_{c}^{0} \rightarrow \Xi^{0} K^{+} K^{-}\right)}{\mathcal{B}\left(\Xi_{c}^{0} \rightarrow \Xi^{-} \pi^{+}\right)} \\
& \quad=0.039 \pm 0.004 \text { (stat.) } \pm 0.002 \text { (syst.) }
\end{aligned}
$$

The measurements of these $\Xi_{c}^{0}$ decay modes, which can only proceed via $W$ exchange together with $s \bar{s}$ production, add to our knowledge of the weak decay of charmed baryons. However, from the amplitude intensities in Fig. 7, we conclude it is unlikely that contributions from these resonant $\Xi_{c}^{0} \rightarrow \Xi^{0} \phi\left(\rightarrow K^{+} K^{-}\right)$decays will correlate to significant event excesses in the $\Xi^{0} K^{-}$reconstruction near $1.95 \mathrm{GeV}$. Only minor cusping to the combinatorial background will be present in that region due to this decay. As a result of the slightly smaller branching fraction via the resonant $\Xi_{c}^{0} \rightarrow \Xi^{0} \phi$ decay, the apparent spin-polarization substructure is diluted due to the nonresonant fraction in this same region. This implies that future excited $\Omega$ searches may promisingly search this region of the $\Xi^{0} K^{-}$invariant mass after the inclusion of these new modes during MC studies. Despite the low statistics of these new modes at Belle, this study via an amplitude analysis provides necessary tools for studying more resonant amplitude features in multibody charmed baryon decays in the forthcoming high-luminosity era.

\section{ACKNOWLEDGMENTS}

We thank the KEKB group for the excellent operation of the accelerator, the KEK cryogenics group for the efficient operation of the solenoid, the KEK computer group and the Pacific Northwest National Laboratory (PNNL) Environmental Molecular Sciences Laboratory (EMSL) computing group for strong computing support, and the National Institute of Informatics and Science Information NETwork 5 (SINET5) for valuable network support. We acknowledge support from the Ministry of Education, Culture, Sports, Science, and Technology (MEXT) of Japan, the Japan Society for the Promotion of Science (JSPS), and the Tau-Lepton Physics Research Center of Nagoya University; the Australian Research Council including Grants No. DP180102629, No. DP170102389, No. DP170102204, No. DP150103061, and No. FT130100303; Austrian Science Fund (FWF); the National Natural Science Foundation of China under Contracts No. 11435013, No. 11475187, No. 11521505, No. 11575017, No. 11675166, and No. 11705209; the Key Research Program of Frontier Sciences, Chinese Academy of Sciences (CAS), Grant No. QYZDJ-SSW-SLH011; the CAS Center for Excellence in Particle Physics (CCEPP); the Shanghai Pujiang Program under Grant No. 18PJ1401000; the Ministry of Education, Youth, and Sports of the Czech Republic under Contract No. LTT17020; the Carl Zeiss Foundation, the Deutsche Forschungsgemeinschaft, the Excellence Cluster Universe, and the VolkswagenStiftung; the Department of Science and Technology of India; the Istituto Nazionale di Fisica Nucleare of Italy; National Research Foundation (NRF) of Korea Grants No. 2016R1D1A1B01010135, No. 2016R1D1A1B02012900, No. 2018R1A2B3003643, No. 2018R1A6A1A06024970, No. 2018R1D1A1B070 47294, 2019K1A3A7A09033840, and No. 2019R1I1 A3A01058933; the Radiation Science Research Institute, Foreign Large-size Research Facility Application supporting project, the Global Science Experimental Data Hub Center of the Korea Institute of Science and Technology Information and KREONET/GLORIAD; the Polish Ministry of Science and Higher Education and the National Science Center; the Ministry of Science and Higher Education of the Russian Federation, Agreement No. 14.W03.31.0026; University of Tabuk Research Grants No. S-1440-0321, No. S-0256-1438, and No. S-0280-1439 (Saudi Arabia); the Slovenian Research Agency; Ikerbasque, Basque Foundation for Science, Spain; the Swiss National Science Foundation; the Ministry of Education and the Ministry of Science and Technology of Taiwan; and the United States Department of Energy and the National Science Foundation. 
[1] R. Chistov et al. (Belle Collaboration), First observation of Cabibbo-suppressed $\Xi_{c}^{0}$ decays, Phys. Rev. D 88, 071103 (2013).

[2] P. A. Zyla et al. (Particle Data Group), Review of Particle Physics, Prog. Theor. Exp. Phys. 2020, 083 C01 (2020).

[3] J. Yelton et al. (Belle Collaboration), Observation of an Excited $\Omega^{-}$Baryon, Phys. Rev. Lett. 121, 052003 (2018).

[4] S. Jia et al. (Belle Collaboration), Search for $\Omega(2012) \rightarrow$ $K \Xi(1530) \rightarrow K \pi \Xi$ at Belle, Phys. Rev. D 100, 032006 (2019).

[5] T. Gutsche, M. A. Ivanov, J. G. Körner, V. E. Lyubovitskij, and P. Santorelli, Polarization effects in the cascade decay $\Lambda_{b} \rightarrow \Lambda\left(\rightarrow p \pi^{-}\right)+J / \Psi\left(\rightarrow l^{+} l^{-}\right)$in the covariant confined quark model, Phys. Rev. D 88, 114018 (2013).

[6] J. Brodzicka et al. (Belle Collaboration), Physics achievements from the Belle Experiment, Prog. Theor. Exp. Phys. 2012, 4D001 (2012).

[7] A. Abashian et al. (Belle Collaboration), The Belle detector, Nucl. Instrum. Methods Phys. Res., Sect. A 479, 117 (2002).
[8] A. Bevan et al. (BABAR, Belle Collaborations), The physics of the B factories, Eur. Phys. J. C 74, 3026 (2014).

[9] T. Kuhr et al. (Belle II Framework Software Group), The Belle II Core Software, Comput. Softw. Big Sci. 3, 1 (2019).

[10] M. Gelb et al. (Belle II Collaboration), B2BII: Data conversion from Belle to Belle II, Comput. Softw. Big Sci. 2, 9 (2018).

[11] J.-F. Krohn et al., Global decay chain vertex fitting at Belle II, Nucl. Instrum. Methods Phys. Res., Sect. A 976, 164269 (2020).

[12] T. Gutsche, M. A. Ivanov, J. G. Körner, V. E. Lyubovitskij, P. Santorelli, and N. Habyl, Semileptonic decay $\Lambda_{b} \rightarrow \Lambda_{c}+$ $\tau^{-}+\overline{\nu_{\tau}}$ in the covariant confined quark model, Phys. Rev. D 91, 074001 (2015); Erratum, Phys. Rev. D 91, 119907 (2015).

[13] D. J. Lange, The EvtGen particle decay simulation package, Nucl. Instrum. Methods Phys. Res., Sect. A 462, 152 (2001).

[14] H. Matevosyan, R. Mitchell, and M. Shepherd, Amptools, https://github.com/mashephe/AmpTools/wiki (2016-2019). 